\title{
Perioperative Radiotherapy is Associated With Improved Survival Among Patients With Synovial Sarcoma: A SEER Analysis
}

\author{
KHATIJA W. NAING, вA, ${ }^{1}$ ARTA M. MONJAZEB, MD, PhD, ${ }^{2}$ CHIN-SHANG LI, PhD, ${ }^{3}$ LI-YUAN LEE, ${ }^{4}$ \\ ANTHONY YANG, MD, ${ }^{1}$ DARIUSZ BORYS, $M{ }^{5},{ }^{5}$ AND ROBERT J. CANTER, MD, MAS ${ }^{1}$ * \\ ${ }^{1}$ Division of Surgical Oncology, Department of Surgery, Davis Medical Center, University of California, Sacramento, California \\ ${ }^{2}$ Department of Radiation Oncology, Davis Medical Center, University of California, Sacramento, California \\ ${ }^{3}$ Division of Biostatistics, Department of Public Health Sciences, University of California Davis, Sacramento, California \\ ${ }^{4}$ Department of Psychology, University of Michigan, Ann Arbor, Michigan \\ ${ }^{5}$ Department of Pathology and Laboratory Medicine, Davis Medical Center, University of California, Sacramento, California
}

Background and Methods: We examined the outcomes of synovial sarcoma (SS) patients in a national database. We identified 1,189 patients from the Surveillance, Epidemiology, and End Results (SEER) database with data on site and extent of surgery. We excluded patients diagnosed before 1990, <18 years, or lacking pathologic confirmation. Using Kaplan-Meier and Cox proportional hazards analyses, we determined predictors of overall (OS) and disease-specific survival (DSS).

Results: The mean age was $41,49.3 \%$ were female, and $82.2 \%$ were white. Radiotherapy (RT) was administered to $57.5 \%$. On multivariable analysis, age at diagnosis, sex, race, anatomic site, SEER summary stage, tumor size, surgery type, and RT predicted OS. Similar predictors of DSS were identified. The hazard ratio (HR) for OS was 0.65 (95\% CI 0.48-0.88) in favor of RT and 0.62 (95\% CI 0.45-0.86) for DSS. Five-year OS improved $8.4 \pm 1.0 \%$ with RT $(P=0.003)$, and five-year DSS improved $7.7 \pm 1.0 \%$ with RT $(P=0.015)$.

Conclusions: In the largest study to date examining the role of RT in synovial sarcoma, we observed that RT was associated with a statistically significant improvement in oncologic outcome among SS patients. These data support the use of RT in the multi-modality treatment of patients with SS.

J. Surg. Oncol. 2015;111:158-164. (C) 2014 Wiley Periodicals, Inc.

KEY WoRDS: synovial sarcoma; radiation therapy; overall survival; disease specific survival; radical surgery

\section{INTRODUCTION}

Soft tissue sarcomas (STS) represent a heterogeneous group of mesenchymal tumors with variable clinical behavior and response to therapy [1]. As a result, the multi-modality treatment of STS is increasingly subtype-specific [2,3]. This often poses problems in treating patients with rare subtypes since extensive data from clinical trials and outcomes studies are often not available to guide rigorous evidence-based management [4].

Synovial sarcoma is one example of a rare subtype of STS, comprising approximately 5-10\% of cases [5]. It commonly occurs in adolescents and young adults, and morphologically often resembles small round cell sarcomas [3,6]. Consequently, it is often grouped with pediatric soft tissue sarcomas such as rhabdomyosarcoma, Ewing's sarcoma, and desmoplastic small round cell sarcoma [5]. However, the age distribution for synovial sarcoma is older than these pediatric sarcomas, and some have questioned whether pediatric STS treatment protocols can be extrapolated to this STS subtype [5].

As is true for other STS, important factors for outcome in synovial sarcoma include tumor size, tumor depth, tumor location, and the ability to achieve a complete resection [7-11]. In general, synovial sarcoma is considered a high-grade sarcoma with poor prognosis, and significant debate has focused on the role of chemotherapy in the multi-modality management of this disease $[5,10,12,13]$. As noted above, many consider cytotoxic chemotherapy a standard component of multimodality therapy $[14,15]$. However, the administration of adjuvant chemotherapy for adult patients with synovial sarcoma remains controversial since definitive evidence from randomized trials is lacking $[4,12,16]$. In addition, due to the potential for overlapping toxicities, the use of radiotherapy (RT) either sequentially or in combination with chemotherapy in an interdigitated approach may limit the ability to administer recommended doses of chemotherapy $[17,18]$. For these reasons, RT is sometimes de-emphasized in the multi-modality therapy for synovial sarcoma patients, and nationally the use of trimodality therapy to treat synovial sarcoma is low $[2,6]$.

The objective of our study was to evaluate the role of RT in the management of synovial sarcoma since this topic has received less evaluation. Although surgical resection in combination with RT is generally considered the backbone of potentially curative therapy for locally advanced STS like synovial sarcoma, some small series have suggested that synovial sarcoma may be resistant to RT [6,19]. Given these concerns, we sought to examine the role of RT in a large national database of synovial sarcoma patients. We hypothesized that synovial sarcoma was a potentially radio-resistant histology and that RT would have a negligible impact on oncologic outcome.

Grant sponsor: National Institutes of Health (UC Davis Paul Calabresi K12 Career Development Award); Grant number: NIH 1K12CA138464-01A2; Grant sponsor: National Center for Advancing Translational Sciences; Grant number: \#UL1 TR000002.

Presented in part at the Connective Tissue Oncology Society 18th Annual Meeting, October 30-November 2, 2013, and the 9th Annual Academic Surgical Congress, February 4-6, 2014.

Conflict of interest: None.

*Correspondence to: Robert J. Canter, MD, Division of Surgical Oncology, Suite 3010, UC Davis Cancer Center, 4501 X Street, Sacramento, CA 95817. Fax: +1-916-703-5267. E-mail: robert.canter@ucdmc.ucdavis.edu

Received 22 July 2014; Accepted 5 August 2014

DOI $10.1002 /$ jso. 23780

Published online 1 September 2014 in Wiley Online Library (wileyonlinelibrary.com). 


\section{METHODS}

Using the Surveillance, Epidemiology, and End Results (SEER) database, we identified all cases of synovial sarcoma (ICD-O-3 codes 9040, 9041, 9042, and 9043) diagnosed from 1990 to 2009. We included patients with pathologic confirmation of the diagnosis and age $\geq 18$ years old. We excluded patients $<18$ years old because of the differences in management of pediatric and adult patients, particularly with respect to the administration of chemotherapy and RT [20,21]. We also excluded patients diagnosed at autopsy, patients with metastatic disease, and patients not undergoing surgical intervention. Our cohort selection process is depicted in Figure 1.

We then abstracted data on patient demographics, primary tumor site, tumor size, stage at diagnosis, receipt of surgical and radiation treatment, marital status, duration of follow-up, vital status, and cause of death. As the standard $\mathrm{T}$ and $\mathrm{N}$ stage information was not consistently available over the course of the study period, we used SEER summary stage as a surrogate variable for stage at diagnosis. We categorized surgical therapy as radical resection (radical surgery and total removal), marginal excision (simple excision), ablation, or biopsy only. Margin status is not abstracted by SEER and thus could not be analyzed. Importantly, data on the administration of chemotherapy are not recorded in SEER and therefore could not be analyzed.

We then performed univariable and multivariable analyses to identify predictors of overall survival (OS) and disease-specific survival (DSS). OS and DSS were estimated using the Kaplan-Meier method. Subgroup analysis was performed testing the association of RT (predictor variable) with both OS and DSS (outcome variable) stratifying by histologic grade and extent of surgical resection. Univariate analyses were compared by the log-rank test. Multivariate analysis was performed using a Cox proportional hazards model, adjusting for age, sex, race, histologic subtype, histologic grade, tumor size, extent of resection, and the administration of RT. Models were also adjusted for multiple comparisons

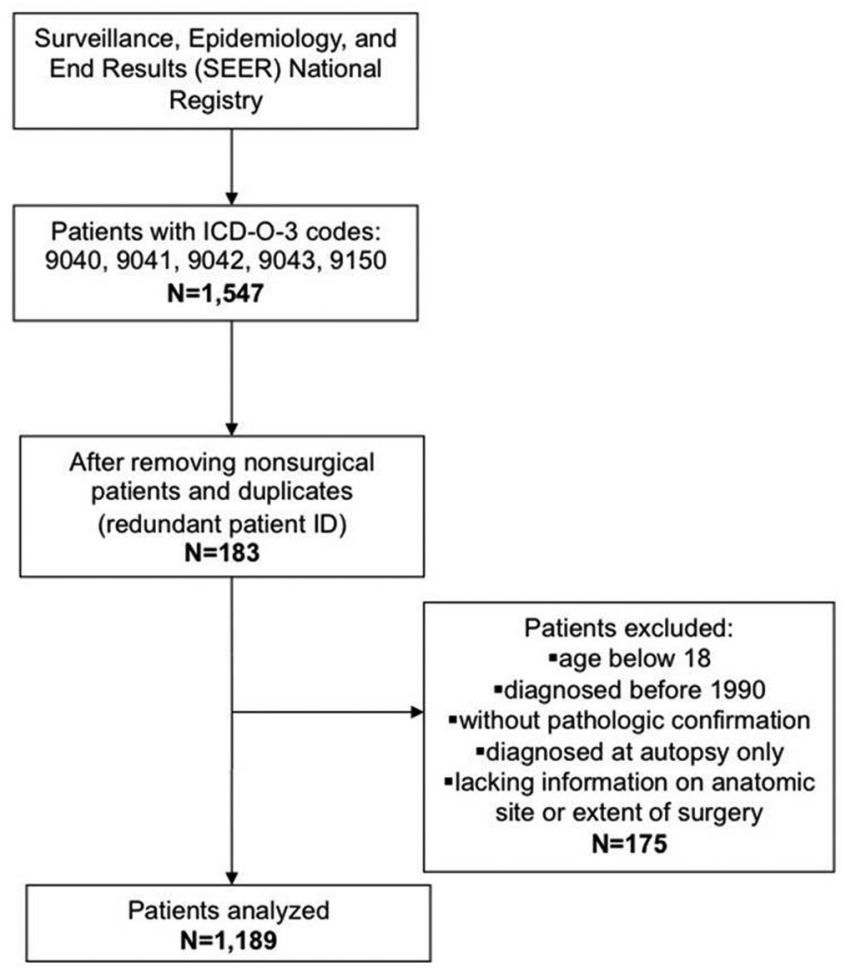

Fig. 1. Schematic flow chart depicting the inclusion and exclusion criteria used to define our study cohort. and subgroups. Analyses were conducted using SAS v9.2 (Cary, NC). Since SEER patient information is de-identified, this study qualified as exempt from UC Davis Institutional Review Board approval.

\section{RESULTS}

\section{Patient and Tumor Characteristics}

1,189 patients met our inclusion criteria (Fig. 1). Baseline patient and tumor characteristics are presented in Table I. The mean age was 41 , $49.3 \%$ were female, and $82.2 \%$ were white. The distribution of histologic grade was: $41.9 \%$ high, $14.0 \%$ intermediate, $2.2 \%$ low, and $41.9 \%$ unknown. Median tumor size was $5.3 \mathrm{~cm}$ (range: $0.3-26.0 \mathrm{~cm}$ ), and the majority of tumors were located in the extremity (67.6\%). $53.2 \%$ of patients were married at the time of diagnosis, while $33.3 \%$ were single, and $9.7 \%$ were separated, divorced, or widowed.

Table I also depicts the extent of surgery performed, including 39.7\% marginal excision and $49.5 \%$ radical surgery. RT was administered to 684 patients $(57.5 \%)$ with 526 patients $(76.9 \%)$ receiving adjuvant RT, 138 patients $(20.1 \%)$ receiving neoadjuvant RT, and 20 patients $(2.9 \%)$

TABLE I. Patient and Tumor Characteristics: Synovial Sarcoma 1990-2009 $(\mathbf{N}=\mathbf{1 , 1 8 9})$

\begin{tabular}{|c|c|}
\hline Variable & Synovial sarcoma $(\mathrm{N}=1,189$ \\
\hline Age at diagnosis (mean $\pm \mathrm{SD})$ & $40.9 \pm 15.6$ \\
\hline \multicolumn{2}{|l|}{ Gender } \\
\hline Female & $586(49.3 \%)$ \\
\hline Male & $603(50.7 \%)$ \\
\hline \multicolumn{2}{|l|}{ Race } \\
\hline White & $977(82.2 \%)$ \\
\hline Black & $126(10.6 \%)$ \\
\hline Asian or Pacific Islander & $66(5.6 \%)$ \\
\hline Other & $20(1.7 \%)$ \\
\hline \multicolumn{2}{|l|}{ Marital status at diagnosis } \\
\hline Married & $633(53.2 \%)$ \\
\hline Separated/divorced/widowed & $115(9.7 \%)$ \\
\hline Single & $396(33.3 \%)$ \\
\hline Unknown & $45(3.8 \%)$ \\
\hline \multicolumn{2}{|l|}{ Histologic grade } \\
\hline Low & $26(2.2 \%)$ \\
\hline Intermediate & $167(14.0 \%)$ \\
\hline High & $498(41.9 \%)$ \\
\hline Unknown & $498(41.9 \%)$ \\
\hline \multicolumn{2}{|l|}{ Tumor location } \\
\hline Extremity & $804(67.6 \%)$ \\
\hline GI/GU/GYN & $15(1.3 \%)$ \\
\hline Head and neck & $95(8.0 \%)$ \\
\hline Retroperitoneal & $3(0.3 \%)$ \\
\hline Thoracic & $76(6.4 \%)$ \\
\hline Trunk & $183(15.4 \%)$ \\
\hline Unknown & $12(1.0 \%)$ \\
\hline Tumor Size, cm (median, range) & $5.3(0.3-26.0)$ \\
\hline \multicolumn{2}{|l|}{ SEER stage } \\
\hline Localized & $782(65.8 \%)$ \\
\hline Regional & $334(28.1 \%)$ \\
\hline Unstaged & $73(6.1 \%)$ \\
\hline Lymph node sampling & $436(36.7 \%)$ \\
\hline Positive lymph nodes & $17(3.9 \%)$ \\
\hline \multicolumn{2}{|l|}{ Radiotherapy } \\
\hline Yes & $684(57.5 \%)$ \\
\hline No & $505(42.5 \%)$ \\
\hline \multicolumn{2}{|l|}{ Type of surgery } \\
\hline Marginal excision & $472(39.7 \%)$ \\
\hline Ablation & $104(8.7 \%)$ \\
\hline Biopsy & $8(0.7 \%)$ \\
\hline Radical resection & $589(49.5 \%)$ \\
\hline Surgery NOS & $16(1.3 \%)$ \\
\hline
\end{tabular}


TABLE II. Patient and Tumor Characteristics Among RT and Non-RT Cohorts

\begin{tabular}{|c|c|c|c|}
\hline Variable & $\mathrm{RT}(\mathrm{N}=684)$ & No RT $(\mathrm{N}=505)$ & $P$-value \\
\hline Age at diagnosis (mean $\pm \mathrm{SD}$ ) & $39.7 \pm 15.0$ & $42.5 \pm 16.2$ & 0.003 \\
\hline Gender & & & 1.000 \\
\hline Female & $337(49.3 \%)$ & $249(49.3 \%)$ & \\
\hline Male & $347(50.7 \%)$ & $256(50.7 \%)$ & \\
\hline Race & & & 0.140 \\
\hline Asian or Pacific Islander & $38(5.6 \%)$ & $28(5.5 \%)$ & \\
\hline Black & $61(8.9 \%)$ & $65(12.9 \%)$ & \\
\hline White & $575(84.1 \%)$ & $402(79.6 \%)$ & \\
\hline Other & $10(1.5 \%)$ & $10(2.0 \%)$ & \\
\hline Histologic grade & & & 0.003 \\
\hline Low & $16(2.3 \%)$ & $10(2.0 \%)$ & \\
\hline Intermediate & $105(15.4 \%)$ & $62(12.3 \%)$ & \\
\hline High & $308(45.0 \%)$ & $190(37.6 \%)$ & \\
\hline Unknown & $255(37.3 \%)$ & $243(48.1 \%)$ & \\
\hline Tumor location & & & $<0.0001$ \\
\hline Extremity & $488(71.3 \%)$ & $316(62.6 \%)$ & \\
\hline GI/GU/GYN & $3(0.4 \%)$ & $12(2.4 \%)$ & \\
\hline Head and neck & $63(9.2 \%)$ & $32(6.3 \%)$ & \\
\hline Retroperitoneal & $2(0.3 \%)$ & $1(0.2 \%)$ & \\
\hline Thoracic & $17(2.5 \%)$ & $60(11.9 \%)$ & \\
\hline Trunk & $107(15.6 \%)$ & $76(15.0 \%)$ & \\
\hline Unknown & $4(0.6 \%)$ & $8(1.6 \%)$ & \\
\hline SEER Stage & & & 0.001 \\
\hline Localized & $466(68.1 \%)$ & $316(61.6 \%)$ & \\
\hline Regional & $191(27.9 \%)$ & $143(28.3 \%)$ & \\
\hline Unstaged & $27(3.9 \%)$ & $46(9.1 \%)$ & \\
\hline Marital status at diagnosis & & & 0.600 \\
\hline Married & $370(54.1 \%)$ & $263(52.1 \%)$ & \\
\hline Separated/divorced/widowed & $68(9.9 \%)$ & $47(9.3 \%)$ & \\
\hline Single & $224(32.8 \%)$ & $172(34.1 \%)$ & \\
\hline Unknown & $22(3.2 \%)$ & $23(4.6 \%)$ & \\
\hline Type of surgery & & & 0.003 \\
\hline Marginal excision & $264(38.6 \%)$ & $208(41.2 \%)$ & \\
\hline Ablation & $69(10.1 \%)$ & $35(6.9 \%)$ & \\
\hline Biopsy & $0(0.0 \%)$ & $8(1.6 \%)$ & \\
\hline Radical resection & $344(50.3 \%)$ & $245(48.5 \%)$ & \\
\hline Surgery NOS & $7(1.0 \%)$ & $9(1.8 \%)$ & \\
\hline $\begin{array}{l}\text { Tumor size, } \mathrm{cm} \\
\text { (median, range) }\end{array}$ & $5.5(0.3-23.0)$ & $5.0(0.5-26.0)$ & 0.326 \\
\hline
\end{tabular}

receiving intra-operative $\mathrm{RT}$. The details of RT treatment, including total dosage, treatment volume, and fractions, were not available for analysis.

\section{Patient and Tumor Characteristics Among RT and Non-RT Cohorts}

A comparison of the patients receiving $\mathrm{RT}$ and those not receiving RT is depicted in Table II. Although patients receiving RT were slightly younger than those not receiving RT $(39.7 \pm 15.0$ years vs. $42.5 \pm 16.2$ years, $P=0.003$ ), there were no other significant differences in demographic factors such as gender, race, or marital status. In contrast, we observed significant differences between the groups for important clinical/pathologic factors such as histologic grade, tumor location, stage of cancer, and type of surgery. For example, RT patients were more likely to have high-grade histology (45.0\% RT vs. $37.6 \%$ non-RT, $P=0.003$ ). In addition, RT patients were more likely to have primary tumors located on the extremity (71.3\% vs. $62.6 \%, P<0.0001)$. Although RT patients were more likely to have localized disease ( 68.1 vs. $61.6 \%, P=0.001)$, rates of regionally advanced disease were comparable (27.9\% RT vs. $28.2 \%$ non-RT). Similarly, ablative procedures were more common among RT patients than non-RT patients $(10.1 \%$ vs. $6.9 \%, P=0.003)$, while marginal excisions (38.6\% vs. $41.2 \%)$ and radical resections $(50.3 \%$ vs. $48.5 \%$ ) were relatively similar. Median tumor size for RT patients was $5.5 \mathrm{~cm}$ (range, $0.3-23.0 \mathrm{~cm}$ ) compared to $5.0 \mathrm{~cm}$ (range, $0.5-$ $26.0 \mathrm{~cm})$ for non-RT patients, a slight difference which was not statistically significant $(P=0.326)$.

\section{Oncologic Outcome}

With a median follow up of 49 months, the five-year OS and DSS among all patients were $69.1 \pm 3.0 \%$ and $72.3 \pm 3.0 \%$, respectively. Median survival was not reached. Among patients receiving RT (Fig. 2A), we observed an approximate $8 \%$ improvement in five-year OS from $64.1 \pm 4.8 \%$ without RT to $72.5 \pm 3.8 \%$ with RT $(P=0.003)$. Similarly, as depicted in Figure $2 \mathrm{~B}$, we observed an approximate $8 \%$ improvement in five-year DSS from $67.7 \pm 4.8 \%$ without RT to $75.4 \pm 3.8 \%$ with RT $(P=0.015)$.

\section{Predictors of Overall and Disease Specific Survival}

On multivariate analysis (Table III), we observed that age at diagnosis $(\mathrm{HR}=1.02,95 \% \mathrm{CI} 1.01-1.03)$ male sex $(\mathrm{HR}=1.63$, CI
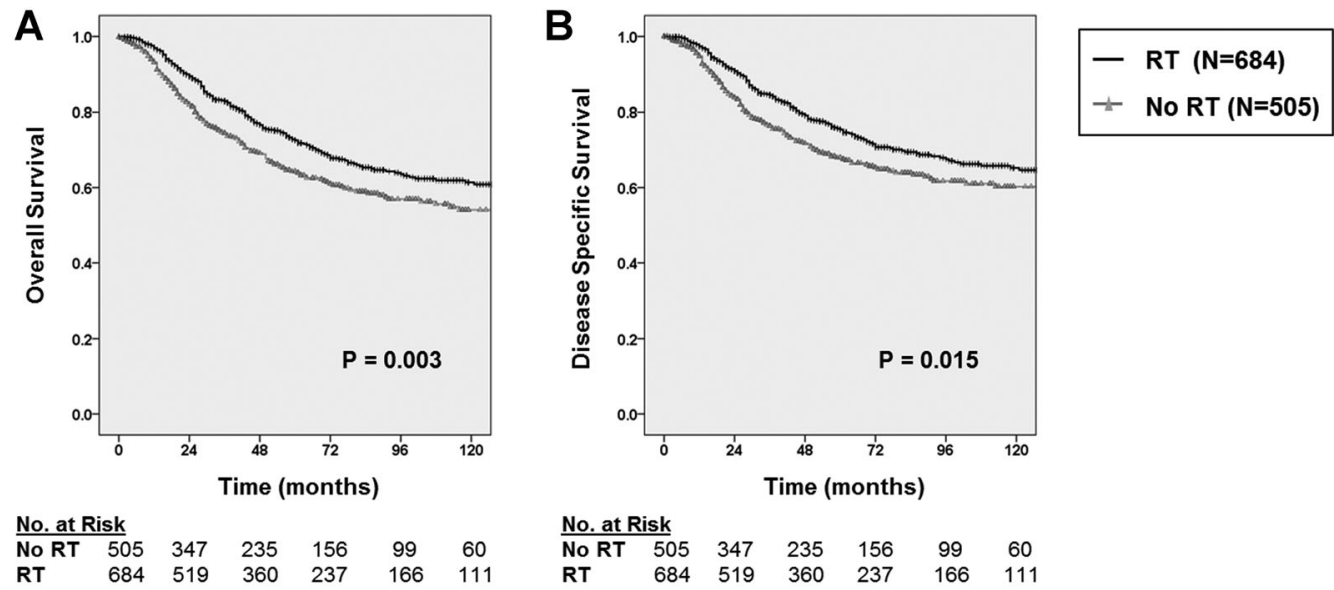

Fig. 2. A: Kaplan-Meier curve depicting overall survival among patients with synovial sarcoma undergoing surgical resection stratified by receipt of radiation therapy $(\mathrm{N}=1,189)$. B: Kaplan-Meier curve depicting disease-specific survival among patients with synovial sarcoma undergoing surgical resection stratified by receipt of radiation therapy. 
TABLE III. Cox Proportional Hazards Multivariable Analysis of Clinicopathologic Variables and Predictors of Overall and Disease-Specific Survival for Synovial Sarcoma Patients Undergoing Surgery $(N=1,189)$

\begin{tabular}{|c|c|c|c|c|}
\hline Variable (predictor) & $\begin{array}{c}\text { Hazard ratio for } \\
\text { overall survival in } \\
\text { synovial sarcoma patients } \\
\text { (95\% confidence interval) }\end{array}$ & $P$-value & $\begin{array}{c}\text { Hazard ratio for } \\
\text { disease-specific } \\
\text { survival in synovial sarcoma } \\
\text { patients (95\% confidence interval) }\end{array}$ & $P$-value \\
\hline Age at diagnosis & $1.02^{*}(1.01-1.03)$ & $<0.001$ & $1.01(0.99-1.02)$ & 0.391 \\
\hline Male & $1.63^{*}(1.22-2.17)$ & $<0.001$ & $1.73^{*}(1.27-2.35)$ & $<0.001$ \\
\hline Race & & 0.002 & & $<0.001$ \\
\hline White & 1.00 (referent) & & 1.00 (referent) & \\
\hline Black & $2.14(1.43-3.22)$ & & $2.43(1.61-3.67)$ & \\
\hline Asian or Pacific Islander & $0.83(0.43-1.59)$ & & $0.66(0.31-1.41)$ & \\
\hline Other & $0.54(0.07-3.94)$ & & $0.66(0.09-4.92)$ & \\
\hline Anatomic site & & $<0.001$ & & $<0.001$ \\
\hline Extremity & 1.00 (referent) & & 1.00 (referent) & \\
\hline Head \& neck & $1.88(1.09-3.24)$ & & $1.71(0.94-3.13)$ & \\
\hline Thoracic & $2.60(1.54-4.38)$ & & $2.68(1.52-4.72)$ & \\
\hline Trunk & $1.80(1.25-2.58)$ & & $1.88(1.28-2.76)$ & \\
\hline Abdominal & $2.20(0.78-6.17)$ & & $2.45(0.87-6.91)$ & \\
\hline Grade & 1.00 (referent) & 0.412 & 1.00 (referent) & 0.226 \\
\hline Low & $0.83(0.34-2.02)$ & & $0.80(0.30-2.13)$ & \\
\hline Intermediate & $0.95(0.41-2.23)$ & & $1.09(0.43-2.76)$ & \\
\hline High & $0.73(0.31-1.70)$ & & $0.78(0.31-1.96)$ & \\
\hline Unknown & & & & \\
\hline SEER stage & & $<0.001$ & & $<0.001$ \\
\hline Localized & 1.00 (referent) & & 1.00 (referent) & \\
\hline Regional & $1.61(1.19-2.19)$ & & $1.83(1.32-2.54)$ & \\
\hline Unstaged & $4.88(2.31-10.3)$ & & $3.73(1.51-9.19)$ & \\
\hline Type of surgery & & $<0.001$ & & $<0.001$ \\
\hline Marginal & 1.00 (referent) & & 1.00 (referent) & \\
\hline Ablation & $0.76(0.46-1.24)$ & & $0.74(0.43-1.28)$ & \\
\hline Biopsy & $44.6(8.91-223.3)$ & & $30.2(3.56-256.8)$ & \\
\hline Radical & $1.19(0.86-1.64)$ & & $1.17(0.83-1.65)$ & \\
\hline Surgery NOS & $6.01(2.06-17.5)$ & & $5.16(1.53-17.4)$ & \\
\hline Radiation & & 0.005 & & 0.003 \\
\hline No & 1.00 (referent) & & 1.00 (referent) & \\
\hline Yes & $0.65(0.48-0.88)$ & & $0.62(0.45-0.86)$ & \\
\hline Tumor size in $\mathrm{cm}$ & $1.13^{*}(1.10-1.16)$ & $<0.001$ & $1.13^{*}(1.10-1.17)$ & $<0.001$ \\
\hline
\end{tabular}

1.22-2.17), African American race $(\mathrm{HR}=2.14,95 \%$ CI 1.43-3.22), tumor location, regionally advanced disease $(\mathrm{HR}=1.61,95 \%$ CI 1.19 $2.19)$, and larger tumor size ( $\mathrm{HR}=1.13,95 \% \mathrm{CI} 1.10-1.16)$ predicted worse OS $(P<0.001)$. Importantly, the administration of RT $(\mathrm{HR}=0.65$, 95\% CI $0.48-0.88)$ was associated with statistically superior OS $(P=0.005)$.

Similarly, we observed that male sex $(\mathrm{HR}=1.73,95 \%$ CI $1.27-$ 2.35), African American race ( $\mathrm{HR}=2.43,95 \%$ CI 1.61-3.67), tumor location, regionally advanced disease $(\mathrm{HR}=1.83,95 \% \mathrm{CI} 1.32-2.54)$, and larger tumor size $(\mathrm{HR}=1.13,95 \%$ CI 1.10-1.17) predicted statistically worse DSS $(P<0.001)$. Notably, RT administration $(\mathrm{HR}=0.62$, 95\% CI $0.45-0.86)$ was again predictive of statistically superior DSS $(P=0.003)$.

\section{Subgroup Analyses}

In order to reduce potential confounding from limited follow up which can correlate with early recurrence/progression following operation [22], we performed subgroup analyses excluding 55 (4.6\% of total) patients with fewer than six months of follow-up time. Using this approach, we observed similar improvements in oncologic outcome with the addition of RT. The five-year OS was improved by $7.3 \%$ following RT $(P=0.01)$, and the five-year DSS in was improved by $6.9 \%$ with RT $(P=0.04)$. We also excluded patients who only received biopsy procedures only $(\mathrm{N}=8)$ since these may have represented patients with unresectable disease. Again, OS and DSS were statistically better with the addition of RT ( $P=0.007$ and $P=0.017$, respectively).

We then analyzed OS and DSS among patients with adverse pathologic features and observed consistent improvement in oncologic outcome among patients receiving RT. For example, as depicted in Figure 3A, among patients with high-grade tumors, five-year OS improved by approximately $13.3 \%$ from $50.3 \pm 8.4 \%$ without RT to $63.6 \pm 6.4 \%$ with RT $(P=0.006)$, and five-year DSS (Fig. 3B) improved by $9.8 \%$ from $56.7 \pm 8.4 \%$ without RT to $66.5 \pm 6.2 \%$ with RT $(P=0.02)$. Similarly, for patients undergoing radical surgery, fiveyear OS (Fig. 4A) improved by approximately $10.5 \%$ from $58.5 \pm 7.4 \%$ without RT to $69.0 \pm 5.8 \%$ with RT $(P=0.008)$, and five-year DSS (Fig. 4B) improved by $10.2 \%$ from $61.7 \pm 7.2 \%$ without RT to $71.9 \pm 5.6 \%$ with RT $(P=0.01)$. Finally, among patients with regionally advanced disease (data not shown), five-year OS improved by approximately $16 \%$ from $42.6 \pm 9.8 \%$ without RT to $58.1 \pm 7.8 \%$ with RT $(P=0.007)$, and five-year DSS improved by $13.4 \%$ from $46.2 \pm 10 \%$ without RT to $59.6 \pm 7.8 \%$ with RT $(P=0.042)$.

\section{DISCUSSION}

We analyzed a large national database of synovial sarcoma patients to identify predictors of oncologic outcomes as well as the impact of RT on OS and DSS. We observed a statistically significant improvement in OS and DSS among patients receiving RT, and this association was 
A

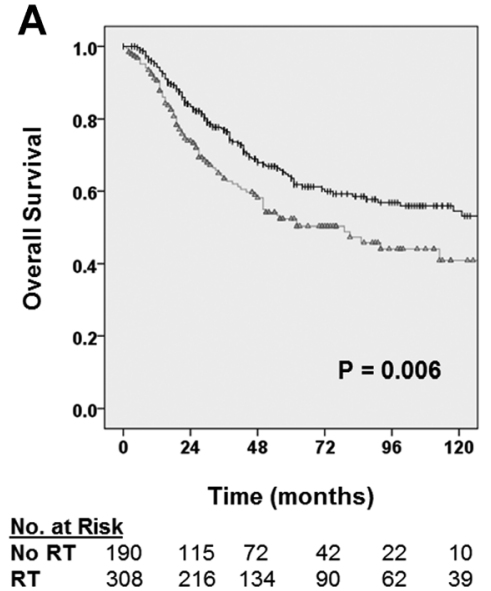

B

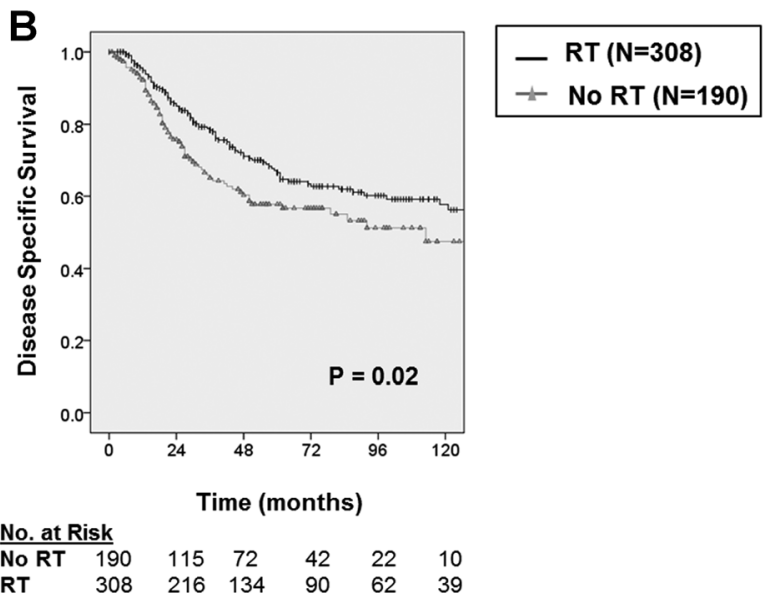

Fig. 3. A: Kaplan-Meier curve depicting overall survival among patients with high-grade tumors stratified by receipt of radiation therapy $(\mathrm{N}=498)$. B: Kaplan-Meier curve depicting disease-specific survival among patients with high-grade tumors stratified by receipt of radiation therapy.

consistently observed in all main subgroups of patients with adverse clinical or pathologic features.

The strengths of our study primarily relate to the large sample size from a population-based database. These factors not only provide greater statistical power to detect clinically meaningful differences but also support the generalizability of our findings. Although there are missing/ absent data in SEER (such as the presence of SYT-SSX gene translocation or the administration of chemotherapy) which we are not able to retrospectively ascertain, the SEER registry nevertheless represents the only comprehensive source of population-based cancer data in the US that includes stage of cancer at the time of diagnosis, cause of death, and patient survival time (http://seer.cancer.gov/).

Furthermore, the characteristics of the patients in this analysis are comparable to those of synovial sarcoma patients reported in the largest single institution series on which current synovial sarcoma-specific outcomes data are based [7,8,10,11,23,24]. For example, similar to Eilber et al. and Ferrari et al., the patients in our cohort were approximately $50 \%$ female, had a median age of 30-40, had maximal tumor dimension in the intermediate range $(5-10 \mathrm{~cm})$, and had the majority of tumors located on the extremity $[7,13]$. Furthermore, similar to these authors, we identified tumor size to be a strong predictor of worse oncologic outcome. We also observed stage at presentation, tumor location, and male sex to be associated with worse oncologic outcome. Somewhat paradoxically, we found radical surgery to be associated with worse oncologic outcome, although we suspect this result is related to the effect of confounding factors which are co-linear with radical surgery, such as high-grade histology and regionally advanced disease.

On multivariate analysis, we found a strong association between RT administration and OS (HR 0.65, $P<0.001$ ) as well as DSS (HR 0.62, $P=0.003)$. In addition, our Kaplan-Meier results show an association between RT and improved OS and DSS for patients receiving radical surgery, suggesting the therapeutic effect of RT is independent of the extent of surgery performed. Similarly, we observed that RT predicted improved OS and DSS in subgroup analyses of patients with high-grade histology and patients with regionally advanced disease, indicating that the association of RT with superior OS and DSS was consistent and reproducible across the subgroups, at least in this population-based dataset. These observations are consistent with the findings of Koshy et al., who
A
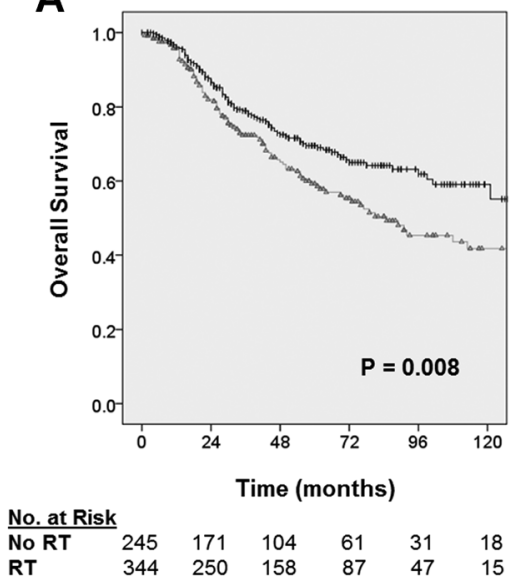

B

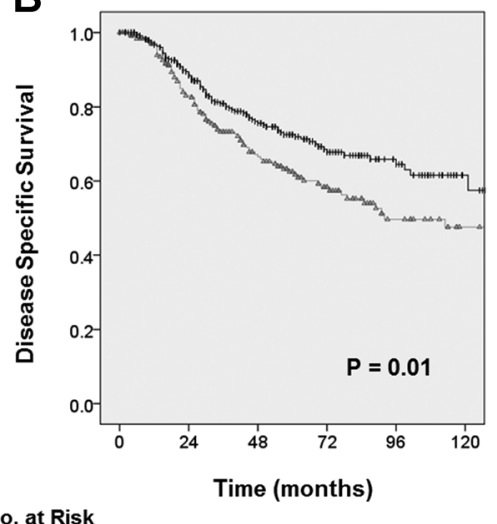

No. at Risk

\begin{tabular}{lllllll}
\hline No RT & 245 & 171 & 104 & 61 & 31 & 18 \\
RT & 344 & 250 & 158 & 87 & 47 & 15
\end{tabular}

Fig. 4. A: Kaplan-Meier curve depicting overall survival among patients receiving radical surgery stratified by receipt of radiation therapy $(\mathrm{N}=589)$. B: Kaplan-Meier curve depicting disease-specific survival among patients receiving radical surgery stratified by receipt of radiation therapy. 
also demonstrated an association between RT and improved overall survival time among heterogeneous extremity STS patients [25]. However, other studies have not reproduced these findings. Yang et al., for example, examined the impact of adjuvant RT among extremity STS patients in a randomized trial and failed to observe a benefit in OS [26]. It is possible, however, that this study was underpowered to detect small, but potentially meaningful, differences in survival, particularly for specific histologic subtypes. Twenty-year follow up from this study, recently reported by Beane et al., again failed to demonstrate an improvement in OS after RT, although patients continued to experience improved local control [27]. In contrast, Schreiber et al. demonstrated (in a retrospective analysis) that RT is associated with improved survival in patients with high grade STS of the extremity undergoing limb-sparing surgery [28].

Although previous studies have evaluated the role of RT in the multimodality management of synovial sarcoma, the results have been equivocal. Some series have demonstrated improved local control and disease-free survival with RT $[8,29]$, while other series have found the opposite or mixed results $[6,10]$. In addition, some authors have suggested that synovial sarcoma may be resistant to RT. Rhomberg, for example, evaluated the radiosensitivity of numerous STS subtypes based on tumor response to definitive RT and subsequent risk of local progression [19]. In this series, synovial sarcomas had one of the lowest response rates to RT with a corresponding higher rate of local tumor progression.

Although relatively few studies have focused on the role of RT in synovial sarcoma, a larger proportion of studies have examined the role of chemotherapy in synovial sarcoma [10,11,13,16,30-32]. Canter et al. constructed a nomogram regarding factors associated with OS and DSS in synovial sarcoma. This analysis observed an early survival benefit to chemotherapy, but did not identify RT as a significant factor for outcome [6]. In contrast, in a study of 102 patients, Al-Hussaini et al. observed that although chemotherapy was routinely used in the management of localized synovial sarcoma, the occurrence of relapse was higher in adult and pediatric patients receiving chemotherapy than among those patients not receiving chemotherapy [16]. Al-Hussaini et al. identified notable differences in the treatment of adult and pediatric patients with a large majority of pediatric patients receiving chemotherapy (in contrast to adult patients) and the opposite relationship for RT. This reinforces the concept that because of the potential for overlapping toxicities, the use of RT either sequentially or in combination with chemotherapy may limit the ability to administer recommended doses of chemotherapy $[17,18]$.

In a study of national practice patterns for STS, Sherman et al. identified synovial sarcoma as a predictor of a higher likelihood to receive adjuvant/neoadjuvant chemotherapy [2]. In addition, these authors demonstrated increasing rates of chemotherapy administration over time among STS patients, including synovial sarcoma patients, with a corresponding decrease in trimodality therapy over time. This study highlights the fact that trimodality therapy can be problematic in adults since the prevalence of severe toxicities may limit any incremental benefits in outcome. Consequently, appropriate selection of patients for combined modality therapy is crucial.

It is important to acknowledge the limitations that come with our study. The SEER database lacks data on important factors such as chemotherapy and status of the surgical margins. In addition, not all patients in the database have complete information. Moreover, in a retrospective study based on local physician and institutional practices, there is likely to be selection bias in terms of which patients received RT. Given the possibility that patients with short follow-up could suffer relapse and death before receiving RT, we performed subgroup analysis excluding patients with fewer than six months of follow up. Our largely unchanged results, therefore, do not support "immortal time bias" in this analysis [22].

We did observe differences in the clinical and pathologic characteristics among patients who received RT and those who did not. Patients receiving RT were slightly younger, had higher-grade tumors, and were more likely to have localized disease, although the percentages of regional disease were similar. Similarly, there was a slight difference in the distribution of surgery with a modest increase in radical surgery among patients receiving RT. Although these imbalances in patient factors may have confounded our results, these factors did not clearly favor the group receiving RT and the improved survival outcomes with RT on both multivariate and multiple subgroup analyses suggest a reproducible and consistent effect.

Despite these limitations, this study is the first population-based analysis of the impact of RT focused on synovial sarcoma patients, and the larger sample size of our study may overcome the limitations of selection bias and lack of generalizability, which are potential weaknesses of single-institution studies. Although the results of our study are appropriately viewed as hypothesis generating, it is unlikely that a histology-based randomized trial examining this question will be performed given the rarity of synovial sarcoma. Therefore, outcomes data such as these are important to inform treatment decisions and help guide avenues for further research.

In summary, although we could not control for the use of chemotherapy in this analysis, RT was associated with a statistically significant improvement in survival among synovial sarcoma patients treated surgically. Improvements in oncologic outcome were most pronounced for patients with adverse pathologic features, and the improvement in OS and DSS was consistent across all subgroups. Although further study is warranted to determine the best multi-modality treatment sequencing, these data support the use of RT in the multidisciplinary approach to patients with synovial sarcoma.

\section{ACKNOWLEDGEMENTS}

This work was supported by funding from the National Institutes of Health (UC Davis Paul Calabresi K12 Career Development Award NIH 1K12CA138464-01A2, R.J. Canter) and the National Center for Advancing Translational Sciences (\#UL1 TR000002, C.S. Li).

\section{REFERENCES}

1. Canter RJ, Beal S, Borys D, et al.: Interaction of histologic subtype and histologic grade in predicting survival for soft-tissue sarcomas. J Am Coll Surg 2010;210:191-198e192.

2. Sherman KL, Wayne JD, Chung J, et al.: Assessment of multimodality therapy use for extremity sarcoma in the United States. J Surg Oncol 2013;109:395-404.

3. Singer S, Demetri GD, Baldini EH, et al.: Management of soft-tissue sarcomas: An overview and update. Lancet Oncol 2000;1:75-85.

4. Katz SC, Brennan MF: Randomized clinical trials in soft tissue sarcoma. Surg Oncol Clin N Am 2010;19:1-11.

5. Eilber FC, Dry SM: Diagnosis and management of synovial sarcoma. J Surg Oncol 2008;97:314-320.

6. Canter RJ, Qin LX, Maki RG, et al.: A synovial sarcoma-specific preoperative nomogram supports a survival benefit to ifosfamidebased chemotherapy and improves risk stratification for patients. Clin Cancer Res 2008;14:8191-8197.

7. Ferrari A, Gronchi A, Casanova M, et al.: Synovial sarcoma: A retrospective analysis of 271 patients of all ages treated at a single institution. Cancer 2004;101:627-634.

8. Guadagnolo BA, Zagars GK, Ballo MT, et al.: Long-term outcomes for synovial sarcoma treated with conservation surgery and radiotherapy. Int J Radiat Oncol Biol Phys 2007;69:1173-1180.

9. Koh KH, Cho EY, Kim DW, et al.: Multivariate analysis of prognostic factors in synovial sarcoma. Orthopedics 2009;32:824.

10. Trassard M, Le Doussal V, Hacene K, et al.: Prognostic factors in localized primary synovial sarcoma: A multicenter study of 128 adult patients. J Clin Oncol 2001;19:525-534.

11. Singer S, Baldini EH, Demetri GD, et al.: Synovial sarcoma: Prognostic significance of tumor size, margin of resection, and mitotic activity for survival. J Clin Oncol 1996;14:1201-1208.

12. Wasif N, Smith CA, Tamurian RM, et al.: Influence of physician specialty on treatment recommendations in the multidisciplinary 
management of soft tissue sarcoma of the extremities. JAMA Surg 2013;148:632-639.

13. Eilber FC, Brennan MF, Eilber FR, et al.: Chemotherapy is associated with improved survival in adult patients with primary extremity synovial sarcoma. Ann Surg 2007;246:105-113.

14. Rosen G, Forscher C, Lowenbraun S, et al.: Synovial sarcoma. Uniform response of metastases to high dose ifosfamide. Cancer 1994;73:2506-2511.

15. Kampe CE, Rosen G, Eilber F, et al.: Synovial sarcoma. A study of intensive chemotherapy in 14 patients with localized disease. Cancer 1993;72:2161-2169.

16. Al-Hussaini H, Hogg D, Blackstein ME, et al.: Clinical features, treatment, and outcome in 102 adult and pediatric patients with localized high-grade synovial sarcoma. Sarcoma 2011;2011:231789.

17. Kraybill WG, Harris J, Spiro IJ, et al.: Phase II study of neoadjuvant chemotherapy and radiation therapy in the management of highrisk, high-grade, soft tissue sarcomas of the extremities and body wall: Radiation Therapy Oncology Group Trial 9514. J Clin Oncol 2006;24:619-625.

18. Pisters PW: Preoperative chemotherapy and split-course radiation therapy for patients with localized soft tissue sarcomas: Home run, base hit, or strike out? J Clin Oncol 2006;24:549-551.

19. Rhomberg W: The radiation response of sarcomas by histologic subtypes: A review with special emphasis given to results achieved with razoxane. Sarcoma 2006;2006:87367.

20. Raney RB: Synovial sarcoma in young people: Background, prognostic factors, and therapeutic questions. J Pediatr Hematol Oncol 2005;27:207-211.

21. Ferrari A, Casanova M, Massimino M, et al.: Synovial sarcoma: Report of a series of 25 consecutive children from a single institution. Med Pediatr Oncol 1999;32:32-37.

22. Park HS, Gross CP, Makarov DV, et al.: Immortal time bias: A frequently unrecognized threat to validity in the evaluation of postoperative radiotherapy. Int J Radiat Oncol Biol Phys 2012;83:1365-1373.

23. Krieg AH, Hefti F, Speth BM, et al.: Synovial sarcomas usually metastasize after $>5$ years: A multicenter retrospective analysis with minimum follow-up of 10 years for survivors. Ann Oncol 2011;22:458-467.

24. Lewis JJ, Antonescu CR, Leung DH, et al.: Synovial sarcoma: A multivariate analysis of prognostic factors in 112 patients with primary localized tumors of the extremity. J Clin Oncol 2000;18:2087-2094.

25. Koshy M, Rich SE, Mohiuddin MM: Improved survival with radiation therapy in high-grade soft tissue sarcomas of the extremities: A SEER analysis. Int J Radiat Oncol Biol Phys 2010;77:203-209.

26. Yang JC, Chang AE, Baker AR, et al.: Randomized prospective study of the benefit of adjuvant radiation therapy in the treatment of soft tissue sarcomas of the extremity. J Clin Oncol 1998; 16:197-203.

27. Beane JD, Yang JC, White D, et al.: Efficacy of adjuvant radiation therapy in the treatment of soft tissue sarcoma of the extremity: 20year follow-up of a randomized prospective trial. Ann Surg Oncol 2014;21:2484-2489.

28. Schreiber D, Rineer J, Katsoulakis E, et al.: Impact of postoperative radiation on survival for high-grade soft tissue sarcoma of the extremities after limb sparing radical resection. Am J Clin Oncol 2012;35:13-17.

29. Shi W, Indelicato DJ, Morris CG, et al.: Long-term treatment outcomes for patients with synovial sarcoma: A 40-year experience at the University of Florida. Am J Clin Oncol 2013;36:83-88.

30. Chen Y, Yang Y, Wang C, et al.: Adjuvant chemotherapy decreases and postpones distant metastasis in extremity stage IIB/III synovial sarcoma patients. J Surg Oncol 2012;106:162-168.

31. Spillane AJ, A'Hern R, Judson IR, et al.: Synovial sarcoma: A clinicopathologic, staging, and prognostic assessment. J Clin Oncol 2000;18:3794-3803.

32. Thompson RC Jr, Garg A, Goswitz J, et al.: Synovial sarcoma. Large size predicts poor outcome. Clin Orthop Relat Res 2000; $18-24$. 